\title{
A Case-Control Study of the 2019 Influenza Vaccine and Incidence of COVID-19 Among Healthcare Workers
}

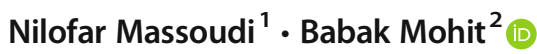 \\ Received: 3 July 2020 / Accepted: 16 November 2020 / Published online: 26 November 2020 \\ (C) Springer Science+Business Media, LLC, part of Springer Nature 2020
}

\begin{abstract}
Purpose The influenza vaccine is essential in reducing the influenza burden, especially among healthcare workers (HCW). Experimental studies suggest both coronaviruses and influenza viruses engage with the angiotensin-converting enzyme 2 (ACE 2) and tetraspanin antibodies, and that ACE 2 tetraspanin antibodies in turn may inhibit both coronavirus and lowpathogenicity influenza A viruses (LP IAV) infections. This study aims to investigate the potential clinical association between receiving the 2019 influenza vaccine and the incidence of COVID-19 among HCW.

Methods We designed a case-control study within a hospital setting in Iran when it became a center for treating COVID-19 patients. We collected data and calculated relevant incidence and associative measures among HCW who had received the 2019 influenza vaccine as compared to HCW who had not received the vaccine.

Results Our total sample size was $261 \mathrm{HCW}$. Of 80 COVID-19 incident cases, three cases had received the influenza vaccine, while 87 of 181 controls had received the vaccine. The odds ratio (OR) and confidence interval (CI) of being vaccinated were 0.04 (95\% CI: 0.01 to 0.14 ) among COVID-19 cases as compared to controls.

Conclusions Significant findings suggest that the 2019 influenza vaccine may have a protective association against COVID-19 among HCW.
\end{abstract}

Keywords COVID-19 $\cdot$ coronavirus $\cdot$ influenza $\cdot$ vaccine $\cdot$ healthcare workers $\cdot$ case-control

\section{Introduction}

The influenza vaccine is both an effective and cost effective way of reducing the burden of influenza, especially among healthcare workers [1-3]. Besides the pathogen specific immunity inducing impact of vaccines, historical precedent hints towards other positive side effects that vaccines may induce. For example, in a randomized controlled trial (RCT) in lowincome countries, measles vaccines administered at 4.5 or

Babak Mohit

bmohit1@alumni.jh.edu

Nilofar Massoudi

massodi@sbmu.ac.ir

1 Anesthesiology Research Center, Department of Anesthesiology, Shahid Beheshti University of Medical Sciences, Tehran, Iran

2 Sleep Disorders Center, Division of Pulmonary and Critical Care Medicine, Department of Medicine, University of Maryland School of Medicine, 100 N. Greene St, 2nd Floor, Baltimore, MD 21201, USA
9 months of age resulted in reduced mortality in the 4.5 to 36 month age group by $30 \%$, while measles death-related events could only account for a $4 \%$ reduction of deaths [4]. In another RCT, the Bacillus Calmette-Guerin (BCG) vaccine reduced neonatal mortality by more than $40 \%$ although tuberculosis (TB), as a cause of death in neonates, is very rare [5]. Clarke and Benn (2015) recommend the study of vaccine's beneficial effect on non-related illnesses, and encourage the reporting of such benefits [6].

Prior in vitro and animal studies suggest that an indirect etiological immunity induction pathway may exist between the influenza vaccine and coronavirus disease 2019 (COVID-19). Animal models have suggested that some subtypes of influenza may lead to a downregulation of angiotensin-converting enzyme 2 (ACE 2), which has protective properties against influenza-induced acute respiratory distress syndrome (ARDS) [7]. ACE 2 has also been suggested as a receptor for viruses from the coronavirus family [8], including severe acute respiratory syndrome coronavirus 2 (SARS-CoV-2), the pathogenic agent of COVID-19 [9]. Furthermore, lab studies report of tetraspanin antibodies that 
inhibit both coronavirus and low-pathogenicity influenza A viruses (LP IAV) infections [10]. This dual inhibition is suggested to be mediated transmembrane proteases such as transmembrane protease serine 2 (TMPRSS2) [10-12], and is reported to interfere with viral proteolytic priming of both LP IAVs and coronaviruses [10].

As of the date of writing this article (on 25 June 2020), global case counts of COVID-19, exceeding 9.5 million confirmed cases, and 488,000 fatalities in 188 countries [13], have affected billions of livelihoods with widespread social and economic ramifications in every continent around the globe. Recent modeling and hypotheses development studies have suggested a potential link between influenza vaccine and COVID-19 [14-17]. Furthermore, case reports have documented the possibility of coinfection with both COVID-19 and influenza [18, 19]. However, to the best of our knowledge, no previous studies have investigated the potential positive clinical side effects between the influenza vaccine and COVID-19 incidence among healthcare workers. Given the large role that $\mathrm{HCW}$ have played globally on the frontlines of treating COVID-19 patients, this study aimed to investigate the potential impact of the 2019 influenza vaccine on the incidence of COVID-19 among a cohort of HCW in a hospital setting during the time when the hospital became a center for treating COVID-19 patients.

\section{Methods}

\section{Study Design}

In order to investigate the potential impact of the 2019 influenza vaccine on the incidence of COVID-19 among HCW in a hospital setting, we designed a single-center, observational case-control study. For this purpose, we submitted the study protocol to the Research Ethics Committee of Shahid Beheshti University of Medical Sciences (Approval ID: IR.SBMU.RETECH.REC.1399 .080 dated 3 May 2020), and sought to collect data on parameters related to the frequency of the 2019 influenza vaccine and the incidence of COVID19, among HCW.

\section{Setting}

Shahid Modarres Specialty Hospital is a 270-bed tertiary care research and teaching facility affiliated with Shahid Beheshti University of Medical Sciences, in Tehran, Iran. It is the only public sector health services facility in northwestern Tehran, and under normal operations, it is home to specialty training fellowships.

The first two cases of COVID-19 were officially reported from Iran on 19 February 2020 [20]. Health authorities informed the hospital of the COVID-19 epidemic on 2
March 2020. This initiated emergency protocol operations of the hospital, including a step-wise increase of COVID-19 inpatient bed allocation to $45,60,90$, and 150 beds on $9,11,13$, and 16 March 2020 respectively. By 20 March 2020, the hospital was serving an average of 140 inpatients with pulmonologist- or test-confirmed COVID-19 per night, and nearly all patients with conditions other than COVID-19 had been transferred. At the end of the data collection of this study, the hospital maintained emergency operations status and was serving an average of 45 inpatients with pulmonologist- or test-confirmed COVID-19 per night.

\section{Study Population and Sample}

The population of this study were the hospital staff composed of 154 medical and 762 nursing, paramedical, and support staff. Emergency operation protocols mandated the presence of all staff members, with the exception of a medically confirmed sick leave.

We used an open source calculator [21] to calculate the minimal required sample size based on the probability of a type I error of alpha $=5 \%$, type II error of beta $=20 \%$ (power $=80 \%$ ), a sample size ratio of 2 , and a hypothesized $20 \%$ difference in proportion of cases with exposed to the vaccine and controls exposed to the vaccine (hypothetical proportion of controls with exposure $30 \%$; hypothetical proportion of cases with exposure: $10 \%)$. This calculation yielded a sample consisting of at least 56 cases and 111 controls. We included all hospital HCW cases with pulmonologist-confirmed COVID-19 in our sample. We applied stratified sampling to select our sample of controls from the hospital staff. We used demographic factors of age, gender, and education as stratification factors to insure that our sample of controls was inclusive. In doing so, trying to assure that our controls were similar to our cases, the first factor we considered was age. Knowing the mean age of our cases, we divided our control in two subsamples (above mean age and below mean age) and sorted each of the two subsamples based on Persian alphabetical order of family name (our randomization factor) and took the first 180 names from each list. After assuring the mean age of this 360-person sample was similar to the mean age of the cases, we used this 360-person sample and repeated the same procedure for gender, and education, to obtain the final control list.

\section{Variables}

Besides demographic factors (age, gender, education, and job type), we sought information on whether the employee had received the 2019 influenza vaccine and the status of their testing for COVID-19, or whether they had been clinically evaluated by a pulmonologist as suspicious for COVID-19 
infection between 10 March 2020 and 10 April 2020 (past 30 days).

\section{Measure of the Influenza Vaccine Intervention}

The 2019 influenza vaccine is described in the Medical Letter on Drugs and Therapeutics [22], and the version used in the hospital was the Influvac sub-unit Tetra, suspension for injection in pre-filled syringe (surface antigen, inactivated) for the 2019/2020 season manufactured by Abbot Labs (UK) [23]. All study participants who received the vaccine were inoculated between September and December 2019 as part of the routine hospital immunization protocol, under which the influenza vaccine is encouraged among hospital staff, but not mandatory. We verified the vaccination status of study participant through employee health records.

\section{Measure of Pulmonologist- or Test-Confirmed COVID- 19 Outcome}

We primarily assessed cases of COVID-19 based on whether they had pulmonologist-confirmed symptoms of fever and dry cough associated with COVID-19 [24]. Due to worldwide shortages of medical supplies, and testing equipment [25], the administration of the SARS-CoV-2 reverse transcription polymerase chain reaction (RT-PCR) test [26] was limited by pulmonologist to only patients that presented clinical symptoms of fever and dry coughing, or HCW who reported workplace unprotected exposure. The sensitivity of the RT-PCR test is reported $93 \%$ while its specificity was $100 \%$ [27]. However, since our samples were collected from nasal swabs, the sensitivity of the test is reported to decrease to $63 \%$ [28].

In order to distinguish between pulmonologist-confirmed and test-confirmed samples, we compiled two datasets. The sample of our first dataset was all study participants based on presentation of clinical symptoms of pulmonologistconfirmed COVID-19 (which were primarily fever and dry cough) [24]. The sample of our second dataset was a subset of study participants who had taken the RT-PCR test for SARS-CoV-2 [24, 26].

\section{Data Collection}

The primary investigator (PI-NM) collected the data on 11 April 2020, and 12 April 2020 using short interviews verified through employee health records. Besides demographic factors (age, gender, education), the PI inquired whether the HCW had received the 2019 influenza vaccine. In addition, the PI sought the status of the HCW testing for COVID-19 using the RT-PCR test, and whether between 10 March 2020 and 10 April 2020 (past 30 days) they had symptoms of fever and dry cough associated with COVID-19 as confirmed by the hospital pulmonologist.

\section{Analysis}

For the total dataset and the confirmed by testing dataset, we calculated the total number the median age, and the mean age of HCW. To create relevant subgroups, we dichotomized exposure to the 2019 influenza vaccine (vaccinated/not vaccinated), as well as each of the demographic variables of age (above/below mean age of sample), gender (male/female), clinical job (yes/no), and each of the three levels of education (high school or below, 2 year or 4 year college degree, graduate degree-yes/no). In order to examine the distribution of the exposure variable (influenza vaccination) among the various demographic groups, we computed the number of $\mathrm{HCW}$ who were vaccinated and not vaccinated, related odds ratio (OR), and 95\% confidence interval (95\% CI) for each stratum.

To examine the main hypothesized relation between influenza vaccination and COVID-19, after obtaining the number of HCW in each stratum, we computed the incidence of HCW who had pulmonologist-confirmed COVID-19 (for the total dataset), or had test-confirmed COVID-19 (for the tested dataset), as well as number of controls for each stratum of each dataset.

For the assessment of the point estimate, the $95 \% \mathrm{CI}$, and the $p$ values of the $\mathrm{OR}$, we employed the epiR package in $\mathrm{R}$ version 4.0.0 (R Foundation for Statistical Computing, Vienna, Austria). After calculating the OR and $95 \% \mathrm{CI}$, we tested the null hypothesis of $\mathrm{OR}=1$ for the main exposure variable and each of the demographic variables. Under our null hypothesis, there would be no significant difference in the incidence of COVID-19 among HCW who had been exposed to the exposure factor (such as the 2019 influenza vaccine) as compared to the incidence of COVID-19 among $\mathrm{HCW}$ who had not been exposed. An OR $<1$ would indicate a protective association, and the entire range of the $95 \% \mathrm{CI}$ would need to be less than 1.00 to produce a significant $p$ value $(p<0.05)$, which would indicate a statistically significant association. We used MS-Excel 2016 (Microsoft Corporation, Seattle, WA, USA) for data gathering, primary analysis, and producing the forest plot of the variables.

\section{Results}

All $n=261 \mathrm{HCW}$ selected for this study volunteered their information. This sample represented $28 \%$ of the population of the hospital staff who presented during the data collection period. We found that of the $261 \mathrm{HCW}, 90 \mathrm{HCW}$ had received the 2019-2020 influenza vaccine. Being female, in a nonclinical job, with a high school or graduate education was associated with a slightly higher odds of being vaccinated, but none of these relations was statistically significant (Table 1). 
Table 1 Characteristics of the sample based on exposure to the Influenza vaccine

\begin{tabular}{|c|c|c|c|c|c|c|c|c|}
\hline & Vaccinated & $\begin{array}{l}\text { Not } \\
\text { vaccinated }\end{array}$ & Total & $\begin{array}{l}\text { Probability } \\
\text { of } \\
\text { vaccination }\end{array}$ & $\begin{array}{l}\text { Odds of } \\
\text { vaccination }\end{array}$ & $\begin{array}{l}\text { OR for } \\
\text { vaccination }\end{array}$ & $95 \% \mathrm{CI}$ & $p$ value \\
\hline $\begin{array}{l}N \\
(\% \text { of total) } \\
\text { Median age (years) }\end{array}$ & $\begin{array}{l}90 \\
(34.48 \%) \\
40\end{array}$ & $\begin{array}{l}171 \\
(65.52 \%) \\
38\end{array}$ & $\begin{array}{l}261 \\
(100.00 \%) \\
39\end{array}$ & 0.34 & 0.53 & & & \\
\hline Mean age (years) & 40.88 & 38.80 & 39.52 & & & & & \\
\hline $\begin{array}{l}\text { Age }>\text { mean age }(39.52 \text { years }) \\
(\% \text { of column } n \text { with age }>39.52)\end{array}$ & $\begin{array}{l}40 \\
(44.44 \%)\end{array}$ & $\begin{array}{l}90 \\
(52.63 \%)\end{array}$ & $\begin{array}{l}130 \\
(49.81 \%)\end{array}$ & 0.31 & 0.44 & 0.72 & 0.43 to 1.21 & 0.21 \\
\hline $\begin{array}{l}\text { Female gender } \\
\text { ( } \% \text { of column } n \text { of females) }\end{array}$ & $\begin{array}{l}43 \\
(47.78 \%)\end{array}$ & $\begin{array}{l}77 \\
(45.03 \%)\end{array}$ & $\begin{array}{l}120 \\
(45.98 \%)\end{array}$ & 0.36 & 0.56 & 1.12 & 0.67 to 1.86 & 0.67 \\
\hline $\begin{array}{l}\text { Clinical job } \\
\text { (\% of column } n \text { with clinical job) }\end{array}$ & $\begin{array}{l}78 \\
(86.67 \%)\end{array}$ & $\begin{array}{l}152 \\
(88.89 \%)\end{array}$ & $\begin{array}{l}230 \\
(88.12 \%)\end{array}$ & 0.34 & 0.51 & 0.81 & 0.38 to 1.76 & 0.60 \\
\hline $\begin{array}{l}\text { Non-clinical job } \\
\text { (\% of column } n \text { with non-clinical job) }\end{array}$ & $\begin{array}{l}12 \\
(13.33 \%)\end{array}$ & $\begin{array}{l}19 \\
(11.11 \%)\end{array}$ & $\begin{array}{l}31 \\
(11.88 \%)\end{array}$ & 0.39 & 0.63 & 1.23 & 0.57 to 2.66 & 0.60 \\
\hline $\begin{array}{l}\text { High school education } \\
\text { (\% of column } n \text { with high school education) }\end{array}$ & $\begin{array}{l}20 \\
(22.22 \%)\end{array}$ & $\begin{array}{l}28 \\
(16.37 \%)\end{array}$ & $\begin{array}{l}48 \\
(18.39 \%)\end{array}$ & 0.42 & 0.71 & 1.46 & 0.77 to 2.77 & 0.25 \\
\hline $\begin{array}{l}\text { College education } \\
\text { ( } \% \text { of column } n \text { with College education) }\end{array}$ & $\begin{array}{l}37 \\
(41.11 \%)\end{array}$ & $\begin{array}{l}84 \\
(49.12 \%)\end{array}$ & $\begin{array}{l}121 \\
(46.36 \%)\end{array}$ & 0.31 & 0.44 & 0.72 & 0.43 to 1.21 & 0.22 \\
\hline $\begin{array}{l}\text { Graduate education } \\
\text { ( } \% \text { of column } n \text { with graduate education) }\end{array}$ & $\begin{array}{l}33 \\
(36.67 \%)\end{array}$ & $\begin{array}{l}59 \\
(34.50 \%)\end{array}$ & $\begin{array}{l}92 \\
(35.25 \%)\end{array}$ & 0.36 & 0.56 & 1.10 & 0.65 to 1.87 & 0.73 \\
\hline
\end{tabular}

In calculating the percentages, $90 / 261=34.48 \%$ of the 261 study participants were vaccinated, therefore yielding a probability of 0.34 and an odds of vaccination of $0.34 /(1-0.34)=0.51$. In controlling for confounders, we found that $43 / 90=47.78 \%$ vaccinated study participants were female, while $77 /$ $171=45.03 \%$ of the non-vaccinated participants were female, and females represented $120 / 261=45.98 \%$ of the total study participants. The probability of vaccination among females was $43 / 120=0.36$, yielding an odds of 0.56 . Therefore, the odds ratio of female symptoms (vs male symptoms, not shown for brevity) was 1.12 , revealing that females were more likely than males to have been vaccinated. However, this was not significant ( $p=0.67)$ as the confidence interval of the odds ratio ranges from as low as 0.67 to as high as 1.76 , where the former figure reveals that females may have been less likely to have been vaccinated. Figures presented for other confounders of clinical job, and education level were calculated similarly, and for clarity, both clinical job and its counterfactual (non-clinical job) are displayed

Table 2 presents the demographic characteristics of our sample. The total number of pulmonologist-confirmed COVID-19 cases was $n=80 \mathrm{HCW}$ (30.65\% of the HCW sample). In our total sample, being older, male, having a non-clinical job, and having a high school education or less were each independently associated with a higher incidence of COVID-19 symptoms; however, only being in the lowest education stratum (having a high school education or less) was significantly associated with developing COVID-19 symptoms (OR $=2.03, p=0.03)$.

A sum of $n=83 \mathrm{HCW}$ (31.8\% of the study sample, and $9.0 \%$ of the population of the hospital staff) were tested for SARS-CoV-2 based on either symptoms or workplace unprotected exposure. The total number of COVID-19 cases based on a positive SARS-CoV-2 RTPCR test was $n=78 \mathrm{HCW}$ (93.98\% of the tested HCW sample). In our tested subsample, being older, female, having a non-clinical job, and having college education were each independently associated with a higher incidence of having a positive RT-PCR test; however, none of these associations was statistically significant. Table 3 displays the characteristics of the subsample tested for SARS-CoV-2.

\section{Vaccination and Symptoms Associated with COVID-19 Among All HCW Surveyed}

Table 4 displays the breakdown of the total $261 \mathrm{HCW}$ based on exposure to the 2019 influenza vaccine and the incidence of pulmonologist-confirmed COVID-19 symptoms. Based on the figures in Table 4, the OR of being vaccinated was 0.04 (95\% CI: 0.01 to 0.14 ) among the case HCW who developed pulmonologist-confirmed COVID-19 symptoms as compared to control HCW who did not develop COVID-19 symptoms. The upper panel of the Fig. 1 depicts this significant association.

\section{Vaccination and Symptoms Associated with COVID-19 Among Tested HCW}

The break-down of the total subsample of $83 \mathrm{HCW}$ tested for SARS-CoV-2 based on exposure to the 2019 influenza vaccine and SARS-CoV-2 RT-PCR test is displayed in Table 5. Based on the figures in Table 5, the OR of being vaccinated was 0.01 (95\% CI: 0.001 to 0.151 ) among the case HCW who had a confirmed positive SARS-CoV-2 RT-PCR test as 


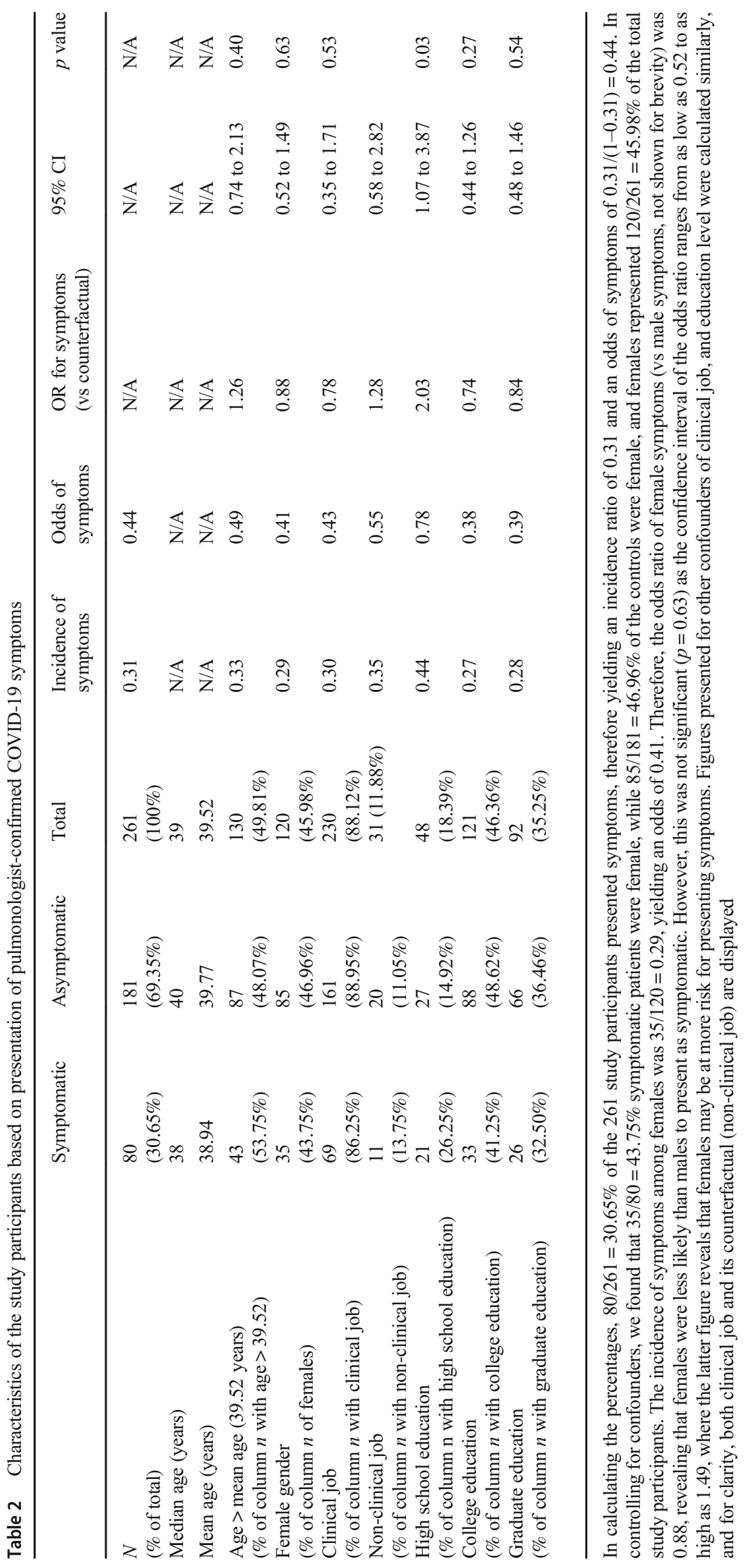




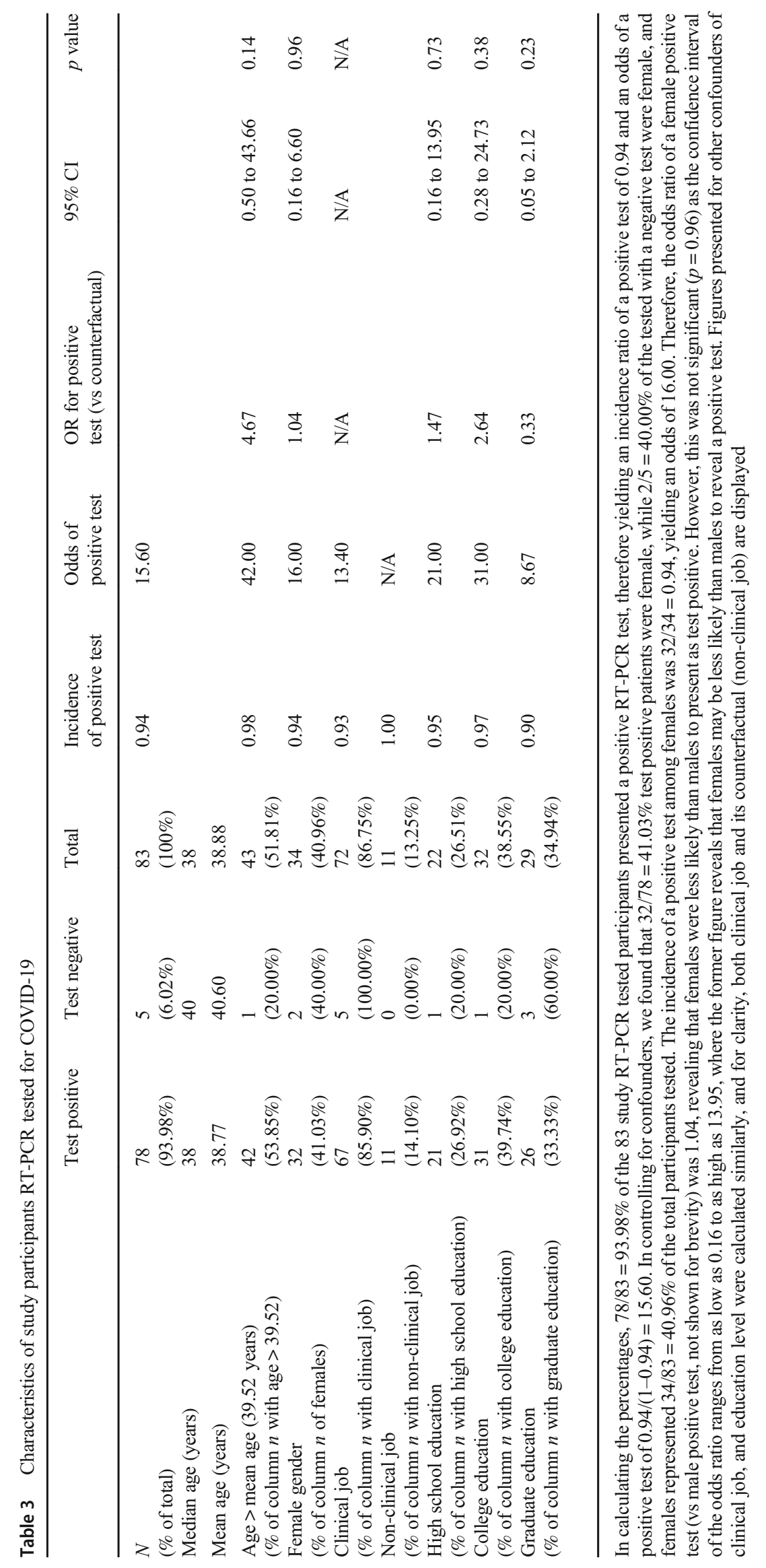


Table 4 Association of 2019 influenza vaccine exposure with pulmonologist-confirmed symptoms of COVID-19

\begin{tabular}{|c|c|c|c|c|c|c|c|c|}
\hline Exposure & Symptomatic & Asymptomatic & Total & $\begin{array}{l}\text { Incidence of } \\
\text { symptoms }\end{array}$ & $\begin{array}{l}\text { Odds of } \\
\text { symptoms }\end{array}$ & OR for symptoms & $95 \% \mathrm{CI}$ & $p$ value \\
\hline $\begin{array}{l}\text { Vaccinated } \\
(\% \text { of column } n)\end{array}$ & $\begin{array}{l}3 \\
(3.75 \%)\end{array}$ & $\begin{array}{l}87 \\
(48.07 \%)\end{array}$ & $\begin{array}{l}90 \\
(34.48 \%)\end{array}$ & 0.03 & 0.03 & 0.04 & 0.01 to 0.14 & $<0.001$ \\
\hline $\begin{array}{l}\text { Not vaccinated } \\
(\% \text { of column } n)\end{array}$ & $\begin{array}{l}77 \\
(96.25 \%)\end{array}$ & $\begin{array}{l}94 \\
(51.93 \%)\end{array}$ & $\begin{array}{l}171 \\
(65.52 \%)\end{array}$ & 0.45 & 0.82 & 23.76 & 7.23 to 78.06 & \\
\hline $\begin{array}{l}n \\
(\% \text { of total } n)\end{array}$ & $\begin{array}{l}80 \\
(30.65 \%)\end{array}$ & $\begin{array}{l}181 \\
(69.35 \%)\end{array}$ & $\begin{array}{l}261 \\
(100.00 \%)\end{array}$ & 0.31 & 0.44 & & & \\
\hline
\end{tabular}

In calculating the percentages, of the 80 people who presented COVID-19 symptoms $3 / 80=3.75 \%$ had been previously vaccinated for influenza Among the 181 symptom free controls, $87 / 181=48.07 \%$ had been previously vaccinated for influenza. The incidence of COVID-19 symptoms among those previously vaccinated for influenza was $3 / 90=0.03$ yielding an odds of $0.03 /(1-0.03)=0.03$. The incidence of COVID-19 symptoms among those previously not vaccinated for influenza was $77 / 171=0.45$ yielding an odds of $0.45 /(1-0.45)=0.82$. Therefore, the odds ratio of developing symptoms among those who were vaccinated (vs those who were not) is $0.03 / 0.82=0.04$, which was highly significant, and with a confidence interval between 0.01 and 0.14 is consistent and may be indicative of a protective association of the influenza vaccine against COVID-19 symptoms. The odds ratio of developing symptoms among those who were not vaccinated (vs those who were) is $0.82 / 0.03=23.76$, which was highly significant, and with a confidence interval between 7.23 and 78.06 is consistent and may be indicative that being unvaccinated is associated with a higher risk of developing COVID-19 symptoms. Overall, of the 261 study participants, $80 / 261=30.65 \%$ developed COVID-19 symptoms, $181 / 261=69.35 \%$ were symptom free, $90 / 261=34.48 \%$ had been vaccinated for influenza, and $171 / 261=65.52 \%$ had not received the influenza vaccine

compared to control HCW who tested negative. The lower panel of Fig. 1 depicts this significant association.

\section{Discussion}

Our results reveal that compared to their control peers, $\mathrm{HCW}$ who tested positive for SARS-CoV-2 or developed clinically confirmed COVID-19 symptoms were significantly less likely to have received the 2019 influenza vaccine. Subgroup analysis, based on socio-demographic characteristics, also confirms this result. For example, within the subgroup, none of 11 non-clinical healthcare workers who tested positive for SARS-CoV-2 received the 2019 influenza vaccine.

There was a single case, who revealed a positive SARSCoV-2 RT-PCR test after the personal-protective equipment of the HCW malfunctioned during the intubation of a COVID19 patient. While the hospital prescribed the HCW to self-

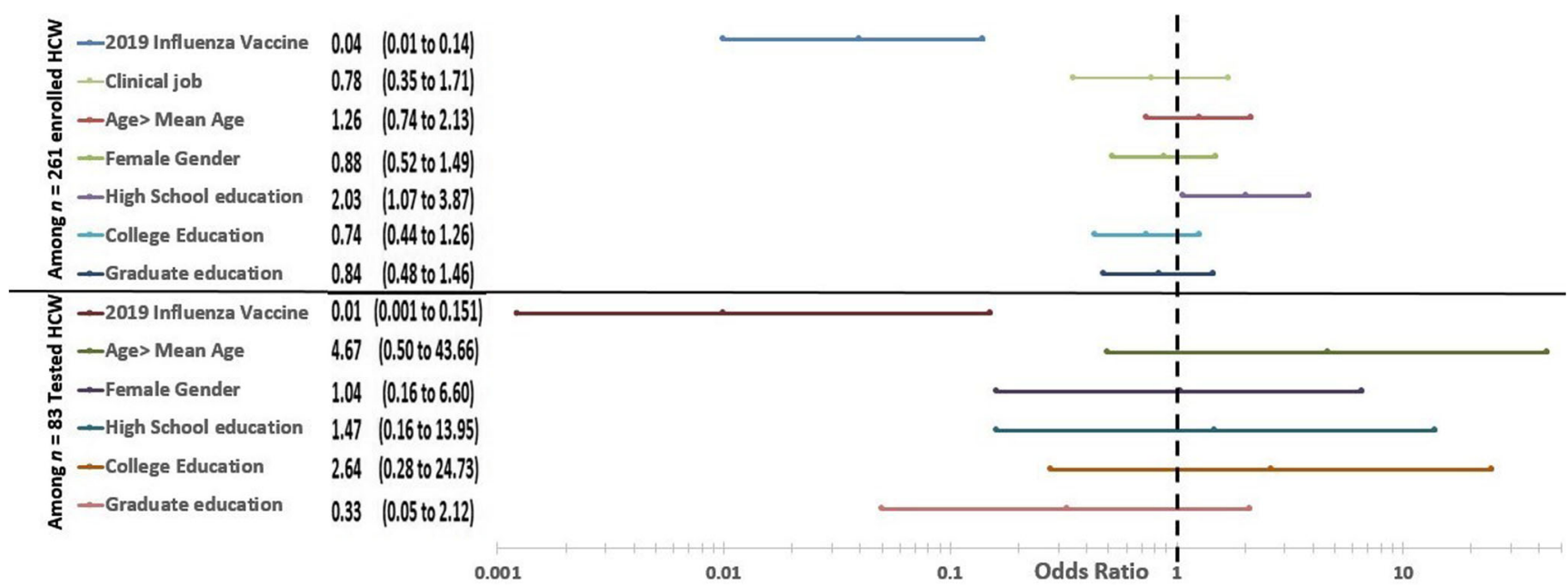

Fig. 1 Forest plot depicting the odds ratio (OR) and 95\% confidence interval $(\mathrm{CI})$ of the association between the incidence of COVID-19 and parameters considered. The horizontal line separates factors in the study. The upper panel is the factors related to the entire sample of healthcare workers (HCW) as enumerated in Table 2 and Table 4, while the lower panel depicts factors related to the subsample of tested HCW enumerated in Table 3 and Table 5. The horizontal axis is a measure of odds ratio (OR) and is on a logarithmic scale. The dotted vertical line is a depiction of $\mathrm{OR}=1$. Points in the middle of the colored lines (each representing the odds ratio associated with one factor) depict the point estimate of the OR (quantified before the parentheses in the legend), while points at the left and right ends depict the extremes of the $95 \%$ confidence interval (quantified within the parentheses in the legend). The colored lines that do not cut through the dotted line indicate statistical significance. The OR reveals a significant association between the incidence of COVID-19 and the 2019 influenza vaccine both among the upper panel $(n=261$ enrolled $\mathrm{HCW})$, and the lower panel $(n=83 \mathrm{HCW})$ 


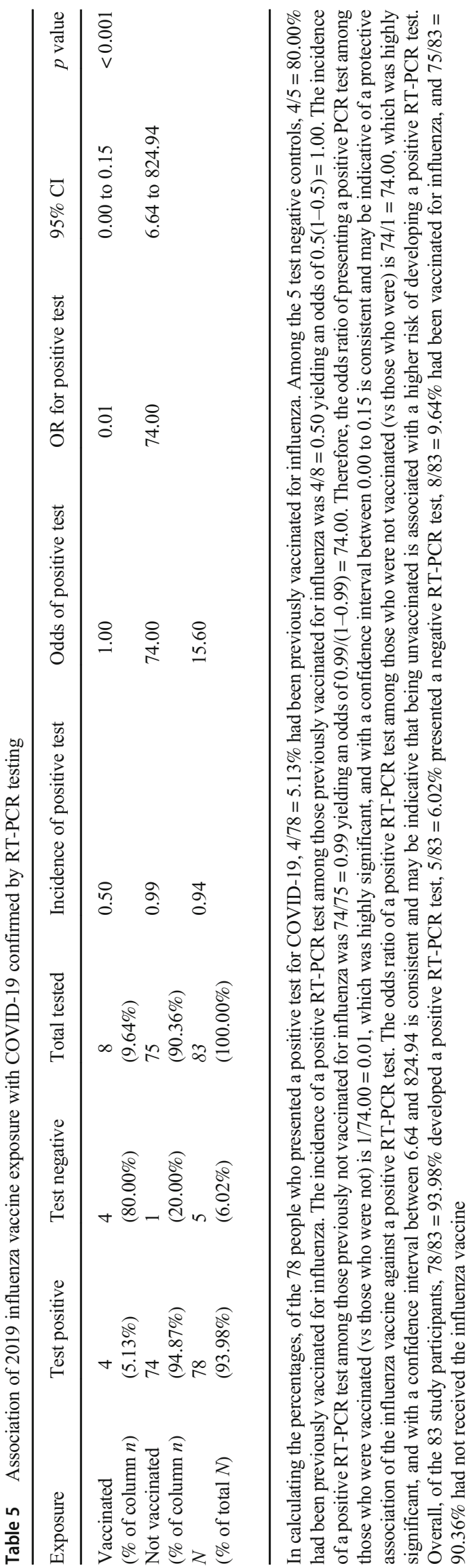

isolate after the positive test, the HCW never reported any symptoms during the 14-day self-isolation period. In reporting this case, since the HCW did not develop symptoms, we counted him as asymptomatic in Table 4, but as test positive in Table 5. In post hoc sensitivity analysis, the significance and the directionality of the OR presented in both Table 4 and Table 5 were robust to changes induced by this one case.

In this hospital (Modarres Hospital), the period for influenza vaccination is between September and December. During this time, staff receive encouraging notices and reminders through billboards, and electronic messaging to receive the vaccine, which is provided free of out of pocket cost. Unlike some healthcare settings in Europe and the USA, however, there is no penalty or restrictions for staff that choose not to receive the vaccine. In our study this may introduce the bias that staff that are better educated or more health conscious may be more likely to receive the vaccine and more likely to have practiced health recommendations that could have prevented their exposure to COVID-19 (such as handwashing). Our controlling for education status was aimed towards managing this potential bias. However, as demonstrated in Table 1, we did not find significant association between the controlled confounders and vaccination status. Furthermore, because of overall increased societal awareness of the COVID-19 pandemic, and the hospital's emergency status, we believe that potential barriers to reporting symptoms were reduced, which reduced the potential for reporting bias.

Since the time this study was conducted, several other studies have also hinted towards a protective association between the influenza vaccine and COVID-19. In a US-based study, Zanetti and colleagues report that influenza vaccination coverage in the elderly population is negatively associated with mortality from COVID-19 and for every $10 \%$ increase in influenza vaccination coverage, there is a $28 \%$ decrease in the rate of mortality from COVID-19 (mortality reduction ratio $(\mathrm{MRR})=0.72,95 \% \mathrm{CI}=0.58-0.89)$ [29]. These results were repeated in Italy where Marin-Hernandez and colleagues report a moderate to strong negative correlation $(r=-0.5874$, $n=21, p=0.0051)$ meaning that where there were higher influenza vaccination rates, less deaths from COVID-19 occurred, and at the regional level each region's percentage of COVID-19 deaths decreased by 0.345 for each unit of percentage of adults $>65$ years old vaccinated against influenza [30]. Also from Italy, Noale et al. report that influenza vaccinations were associated with a decreased probability of a SARS-CoV-2 positive test in the younger participants $(\mathrm{OR}=0.85,95 \% \mathrm{CI} 0.74-0.98)$. In yet another study of US firefighters, Caban-Martinez and colleagues report that none of the firefighters/paramedics who tested positive for SARSCoV-2 antibodies reported receipt of the annual influenza vaccine compared with firefighters/paramedics who tested negative for SARS-CoV-2 antibodies $(0.0 \%$ vs $21.0 \%$; $p=$ 
0.027) [31]. In a risk model of developing COVID-19 symptoms based on various health risks from the USA, Jehi and colleagues report that influenza vaccination was associated with a lower risk of COVID-19 infection and influenza vaccination rate was $93.9 \%$ among 5940 of non-COVID-19 controls vs $6.1 \%$ among 384 COVID-19 positive cases $(p<0.001)$ [32].

Several studies have hinted to possible mechanisms in which the influenza vaccine may interfere with the pathogenicity of SARS-CoV-2. Earnest et al. [10] have argued that both coronaviruses and low-pathogenicity influenza A viruses (LP IAVs) depend on target cell proteases to cleave their viral glycoproteins and prime them for virus-cell membrane fusion. Several proteases cluster into tetraspanin-enriched microdomains (TEMs), suggesting that TEMs are preferred virus entry portals. Their work reveals that tetraspanin antibodies inhibited $\mathrm{CoV}$ and $\mathrm{LP}$ IAV infections. Their findings suggest that TEMs are exploited by coronaviruses and LP IAVs for appropriate coengagement with cell receptors and proteases. On the other hand, Chakraborty et al. [33], while explaining the role of convalescent serum therapy antibodies in the pathogenesis of COVID-19, have demonstrated that adults with PCR-diagnosed COVID-19 produce IgG antibodies with a specific molecular structure that is characterized by reduced sugars (fucosylation), in the structure of IgG antibody. They reveal that the antibodies of these adults had less fucosylation as compared with SARS-CoV-2seropositive children and relative to adults with symptomatic influenza virus infections. The authors posit that it is unclear whether $\mathrm{IgG}$ fucosylation is reduced prior to infection in individuals who are susceptible to COVID-19 disease or whether this modification is triggered by infection itself, and argue that if fucosylation is reduced prior to infection, this could be a marker of susceptibility to COVID-19. Finally, they note that influenza infection does not trigger fucosylation of IgG. Data collected by Ziegler et al. [34] suggests that SARS-CoV-2 could exploit interferon-driven upregulation of ACE2, a tissueprotective mediator during lung injury, to enhance infection and the authors argue that influenza infection also induces broader expression of ACE2 in upper airway epithelial cells. Finally, in a short overview of possible mechanisms, Eldanasory and colleagues [35] note that influenza vaccination could act as a non-specific immune stimulator in patients with COVID-19, leading to early activation of the immune system to attack SARS-CoV-2 before invading cells, and stimulation of the immune system by influenza vaccines could occur through early activation of the immune system by influenza vaccine which facilitate early detection of SARS-CoV-2. They highlight evidence that influenza vaccine keeps the immune system active through Toll-Like Receptor 7 [32]. Toll-Like Receptor 7 is an important binding of single-stranded RNA respiratory viruses, including SARS-CoV-2 [36]. On the other hand, an actual influenza $A$ infection may upregulate pulmonaryACE2 receptors and leading to increased SARS-CoV-2 infection [37].

In controlling for other bio-immunological factors, we did consider and gather data on factors that may have been immunological confounders. However, the incidence of each of the individual confounders was negligible. A total nine cases had potential immunological confounders. This included one smoker, one with a history of splenectomy, two with a history of asthma, one with obesity (BMI $>40$ ), three diabetics, and one immunosuppressed person who was receiving IV-Ig therapy. On the other hand, 11 controls had potential immunological confounders. This included two persons with a history of pneumonia in the past 3 months, one person with active COPD, one pregnant woman, three smokers, two diabetics, and two persons who were receiving corticosteroids of which one of them was a victim of chemical warfare from the IranIraq war in the 1980s. We concluded that a total of nine cases and 11 controls with such a diversity of factors is too small of a sample size and therefore underpowered to be able draw any significant conclusions.

Our study has several limitations. The most important limitation is that, due to limited testing availability, our ability to detect asymptomatic cases of COVID-19 among HCW was limited. We managed this limitation by reporting and analyzing pulmonologist-and test-confirmed cases separately. However, given the inherent insufficient sample size and lack of power of the results of Table 5, we posit that the results of Table 4 are likely to be better illustrative of the association between influenza vaccination and COVID-19 than Table 5 .

The sample of our study is limited to HCW from one hospital in one country setting. Therefore, the protective benefits our results suggest may not be generalizable to $\mathrm{HCW}$ in the same country, or HCW in other health systems, and the general population. Indeed, countries with far higher influenza vaccination rates [38] have reported higher incidences of COVID-19 cases and mortality [13]. On the other hand, however, countries with higher BCG vaccination rates have also seen lower incidences of COVID-19 infection and mortality [39]. Given that the entire population of our study was also vaccinated with the BCG vaccine, the results of our study may suggest the necessity of the synergistic effect of both the BCG and the influenza vaccines in order to achieve the protective association against COVID-19.

Our study is the first study to focus on the potential sidebenefits of the influenza 2019 vaccine, and COVID-19 among $\mathrm{HCW}$. Future prospective studies may benefit from our results in observing whether these results are repeatable in observational and experimental studies in other locales, among both $\mathrm{HCW}$ and the general population. Furthermore, improvement of our understanding of the etiological pathways of the 
pathogenicity of both SARS-CoV-2 and influenza may lead to future vaccine development and improvement. In conclusion, our significant results lead us to conclude that the 2019 influenza vaccine may have a protective association against COVID-19 among HCW.

Acknowledgments We dedicate this article to the committed healthcare workers around the globe, who through their personal sacrifices reduced the burden of COVID-19. We are especially thankful of the staff of Shahid Modarres Specialty Hospital whose sacrifices we witnessed firsthand, and who trusted us with their data, which made this article possible.

Author Contributions NM developed the study concept, collected the data, supervised the study, and critically revised the manuscript, and read and approved the final manuscript.

BM developed the study protocol, performed statistical analysis, analyzed and interpreted the data, drafted the original manuscript, and read and approved the final manuscript.

Data availability Study protocol, statistical code, and the datasets used and analyzed during the current study are available from the corresponding author (bmohit1@alumni.jh.edu) on reasonable request.

\section{Compliance with Ethical Standards}

Competing Interests The authors declare that they have no competing interests.

\section{References}

1. Fiore AE, Bridges CB, Cox NJ. Seasonal influenza vaccines. Curr Top Microbiol Immunol. 2009;333:43-82. https://doi.org/10.1007/ 978-3-540-92165-3 3.

2. Ting EEK, Sander B, Ungar WJ. Systematic review of the costeffectiveness of influenza immunization programs. Vaccine. 2017;35(15):1828-43. https://doi.org/10.1016/j.vaccine.2017.02. 044.

3. Imai C, Toizumi M, Hall L, Lambert S, Halton K, Merollini K. A systematic review and meta-analysis of the direct epidemiological and economic effects of seasonal influenza vaccination on healthcare workers. PLoS One. 2018;13(6):e0198685. https://doi. org/10.1371/journal.pone.0198685.

4. Aaby P, Martins CL, Garly ML, Bale C, Andersen A, Rodrigues A et al. Non-specific effects of standard measles vaccine at 4.5 and 9 months of age on childhood mortality: randomised controlled trial. BMJ (Clinical research ed). 2010;341:c6495. doi:https://doi.org/10. 1136/bmj.c6495

5. Aaby P, Roth A, Ravn H, Napirna BM, Rodrigues A, Lisse IM, et al. Randomized trial of BCG vaccination at birth to low-birthweight children: beneficial nonspecific effects in the neonatal period? J Infect Dis. 2011;204(2):245-52. https://doi.org/10.1093/ infdis/jir240.

6. Clarke MS, Benn CS. Unusual positive effects from vaccines need to be reported - they represent a resource that could lead to new treatment strategies. Vaccine. 2015;33(28):3162-3. https://doi.org/ 10.1016/j.vaccine.2015.04.076.

7. Yang P, Gu H, Zhao Z, Wang W, Cao B, Lai C, et al. Angiotensinconverting enzyme 2 (ACE2) mediates influenza H7N9 virusinduced acute lung injury. Sci Rep. 2014;4:7027. https://doi.org/ 10.1038/srep07027.
8. Ge XY, Li JL, Yang XL, Chmura AA, Zhu G, Epstein JH, et al. Isolation and characterization of a bat SARS-like coronavirus that uses the ACE2 receptor. Nature. 2013;503(7477):535-8. https:// doi.org/10.1038/nature12711.

9. Hoffmann M, Kleine-Weber H, Schroeder S, Kruger N, Herrler T, Erichsen $\mathrm{S}$ et al. SARS-CoV-2 Cell Entry Depends on ACE2 and TMPRSS 2 and Is Blocked by a Clinically Proven Protease Inhibitor. Cell. 2020;181(2):271-80.e8. doi:https://doi.org/10. 1016/j.cell.2020.02.052.

10. Earnest JT, Hantak MP, Park JE, Gallagher T. Coronavirus and influenza virus proteolytic priming takes place in tetraspaninenriched membrane microdomains. J Virol. 2015;89(11):6093104. https://doi.org/10.1128/jvi.00543-15.

11. Wambier CG, Goren A. Severe acute respiratory syndrome coronavirus 2 (SARS-CoV-2) infection is likely to be androgen mediated. J Am Acad Dermatol. 2020;83(1):308-9. https://doi.org/10. 1016/j.jaad.2020.04.032.

12. Wambier CG, Goren A, Vaño-Galván S, Ramos PM, Ossimetha A, $\mathrm{Nau}$ G, et al. Androgen sensitivity gateway to COVID-19 disease severity. Drug Dev Res. 2020;81:771-6. https://doi.org/10.1002/ ddr. 21688 .

13. Dong E, Du H, Gardner L. An interactive web-based dashboard to track COVID-19 in real time. Lancet Infect Dis. 2020;20:533-4. https://doi.org/10.1016/S1473-3099(20)30120-1.

14. Gurwitz D. Angiotensin receptor blockers as tentative SARS-CoV2 therapeutics. Drug Dev Res. 2020;81:537-40. https://doi.org/10. 1002/ddr.21656

15. Koo JR, Cook AR, Park M, Sun Y, Sun H, Lim JT, et al. Interventions to mitigate early spread of SARS-CoV-2 in Singapore: a modelling study. Lancet Infect Dis. 2020;20:67888. https://doi.org/10.1016/s1473-3099(20)30162-6.

16. Li Q, Tang B, Bragazzi NL, Xiao Y, Wu J. Modeling the impact of mass influenza vaccination and public health interventions on COVID-19 epidemics with limited detection capability. Math Biosci. 2020;325:108378. https://doi.org/10.1016/j.mbs.2020. 108378.

17. Salem ML, El-Hennawy D. The possible beneficial adjuvant effect of influenza vaccine to minimize the severity of COVID-19. Med Hypotheses. 2020;140:109752. https://doi.org/10.1016/j.mehy. 2020.109752.

18. Ozaras R, Cirpin R, Duran A, Duman H, Arslan O, Bakcan Y, et al. Influenza and COVID-19 co-infection: report of 6 cases and review of the literature. J Med Virol. 2020;92:2657-65. https://doi.org/10. $1002 /$ jmv.26125.

19. Konala VM, Adapa S, Naramala S, Chenna A, Lamichhane S, Garlapati PR, et al. A case series of patients coinfected with influenza and COVID-19. Journal of investigative medicine high impact case reports. 2020;8:2324709620934674. https://doi.org/10.1177/ 2324709620934674.

20. Ministry of Health Treatment and Medical Education IR Iran. Chair of Public Relations and Media of the Ministry of Health: Primary test results of two suspected cases of coronavirus have been reported to be positive / Confirmatory tests are being completed. [in Persian] 2020. https://bit.ly/3a3jYC9. Accessed April 14, 2020.

21. Sullivan KM. Pezzullo JC. Dean AG: Mir RA. Open source statistics for public health; 2013. https://www.openepi.com/SampleSize/ SSCohort.htm

22. The Medical Letter. Influenza vaccine for 2019-2020. Jama. 2019;323(1):84-5. https://doi.org/10.1001/jama.2019.18016.

23. Abbot Labs. PACKAGE LEAFLET: INFORMATION FOR THE USER. Influvac sub-unit Tetra, suspension for injection in prefilled syringe. Influenza vaccine (surface antigen, inactivated) 2019/2020 season. 2019. https://www.medicines.org.uk/emc/files/ pil.9381.pdf.

24. World Health Organization (WHO). Global Surveillance for human infection with novel coronavirus (2019-nCoV) - Interim guidance. 
2020. https://apps.who.int/iris/bitstream/handle/10665/330857/ WHO-2019-nCoV-SurveillanceGuidance-2020.3-eng.pdf. Accessed 2 March 2020.

25. Nicoli F, Gasparetto A. Italy in a time of emergency and scarce resources: the need for embedding ethical reflection in social and clinical settings. The Journal of clinical ethics. 2020;31(1):92-4.

26. World Health Organization (WHO). Laboratory testing for coronavirus disease (COVID-19) in suspected human cases. 2020. https:// www.who.int/publications-detail/laboratory-testing-for-2019novel-coronavirus-in-suspected-human-cases-20200117.

27. Corman VM, Landt O, Kaiser M, Molenkamp R, Meijer A, Chu DK et al. Detection of 2019 novel coronavirus (2019-nCoV) by real-time RT-PCR. Euro Surveill. 2020;25(3). doi:https://doi.org/ 10.2807/1560-7917.ES.2020.25.3.2000045.

28. Wang W, Xu Y, Gao R, Lu R, Han K, Wu G, et al. Detection of SARS-CoV-2 in different types of clinical specimens. Jama. 2020. https://doi.org/10.1001/jama.2020.3786.

29. Zanettini C, Omar M, Dinalankara W, Imada EL, Colantuoni E, Parmigiani $G$ et al. Influenza vaccination and COVID19 mortality in the USA. medRxiv : the preprint server for health sciences. 2020. doi:https://doi.org/10.1101/2020.06.24.20129817.

30. Marín-Hernández D, Schwartz RE, Nixon DF. Epidemiological evidence for association between higher influenza vaccine uptake in the elderly and lower COVID-19 deaths in Italy. J Med Virol. 2020. https://doi.org/10.1002/jmv.26120.

31. Caban-Martinez AJ, Schaefer-Solle N, Santiago K, LouzadoFeliciano P, Brotons A, Gonzalez M, et al. Epidemiology of SARS-CoV-2 antibodies among firefighters/paramedics of a US fire department: a cross-sectional study. Occup Environ Med. 2020;77:857-61. https://doi.org/10.1136/oemed-2020-106676.

32. Jehi L, Ji X, Milinovich A, Erzurum S, Rubin BP, Gordon S, et al. Individualizing risk prediction for positive coronavirus disease 2019 testing: results from 11,672 patients. Chest. 2020;158(4): 1364-75. https://doi.org/10.1016/j.chest.2020.05.580.
33. Chakraborty S, Edwards K, Buzzanco AS, Memoli MJ, Sherwood $\mathrm{R}$, Mallajosyula V et al. Symptomatic SARS-CoV-2 infections display specific IgG Fc structures. medRxiv : the preprint server for health sciences. 2020. doi:https://doi.org/10.1101/2020.05.15. 20103341.

34. Ziegler CGK, Allon SJ, Nyquist SK, Mbano IM, Miao VN, Tzouanas CN et al. SARS-CoV-2 receptor ACE2 is an interferonstimulated gene in human airway epithelial cells and is detected in specific cell subsets across tissues. Cell. 2020;181(5):1016-35.e19. doi:https://doi.org/10.1016/j.cell.2020.04.035.

35. Eldanasory OA, Rabaan AA, Al-Tawfiq JA. Can influenza vaccine modify COVID-19 clinical course? Travel Med Infect Dis. 2020;37:101872. https://doi.org/10.1016/j.tmaid.2020.101872.

36. Poulas K, Farsalinos K, Zanidis C. Activation of TLR7 and innate immunity as an efficient method against COVID-19 pandemic: imiquimod as a potential therapy. Front Immunol. 2020;11:1373. https://doi.org/10.3389/fimmu.2020.01373.

37. Hui KPY, Cheung MC, Perera R, Ng KC, Bui CHT, Ho JCW, et al. Tropism, replication competence, and innate immune responses of the coronavirus SARS-CoV-2 in human respiratory tract and conjunctiva: an analysis in ex-vivo and in-vitro cultures. Lancet Respir Med. 2020;8(7):687-95. https://doi.org/10.1016/S2213-2600(20) 30193-4.

38. Organization for Economic Cooperation and Development (OECD). Influenza vaccination rates. 2018. https://data.oecd.org/ healthcare/influenza-vaccination-rates.htm.

39. Miller A, Reandelar MJ, Fasciglione K, Roumenova V, Li Y, Otazu $\mathrm{GH}$. Correlation between universal BCG vaccination policy and reduced morbidity and mortality for COVID-19: an epidemiological study. medRxiv 2020032420042937. 2020. doi:https://doi.org/ $10.1101 / 2020.03 .24 .20042937$.

Publisher's Note Springer Nature remains neutral with regard to jurisdictional claims in published maps and institutional affiliations. 